\title{
Thoracic radiotherapy and concurrent almonertinib for unresectable stage III EGFR-mutated non-small-cell lung cancer: a phase 2 study
}

Lucheng ZHU ${ }^{1}$, Changlin Zou ${ }^{2}$, Zhanchun Zhang ${ }^{3}$, Jianfang Wang ${ }^{4}, \mathrm{Li}_{\text {Yang }}{ }^{5}$, Chuangzhou Rao ${ }^{6}$, Zhiping Yang ${ }^{7}$, Jiafeng Liang ${ }^{1}$, Bing $\mathrm{Xia}^{1,8^{*}}$ and M. A. Shenglin ${ }^{1,9^{*}}$

\begin{abstract}
Background: Concurrent chemo-radiotherapy remains the standard treatment in unresectable stage III non-smallcell lung cancer (NSCLC) patients. Several studies have shown a potential value of concurrent epidermal growth factor receptor-tyrosine kinase inhibitor (EGFR-TKI) with thoracic radiotherapy in EGFR-mutated population, but a high risk of radiation pneumonitis raised a major concern. This study intends to explore the safety and efficacy of concurrent almonertinib, a new third-generation EGFR-TKI, with radiotherapy in locally advanced EGFR-mutated NSCLC patients.

Methods: Locally advanced NSCLC patients harboring sensitive EGFR mutation will be included in this study. A radiotherapy plan will be made for each patient before treatment, and the lung V20 will be calculated. Patients with lung V20 $\geq 28 \%$ were enrolled in induction group (arm A), which almonertinib was given for 2 months followed by concurrent radiotherapy; patients with lung V $20<28 \%$ were enrolled in concurrent group (arm B), which almonertinib was given concurrent with thoracic radiotherapy. The primary endpoint is the incidence of grade $\geq 3$ radiation pneumonitis within 6 months post-radiotherapy, and the secondary endpoints are local control rate, progression-free survival, and overall survival.

Discussion: The safety and efficacy of third-generation EGFR-TKI concurrent with thoracic radiotherapy in locally advanced EGFR-mutated NSCLC is still unknown. We propose to conduct this phase 2 study evaluating the safety especially the radiation pneumonitis within 6 months post-radiotherapy. This trial protocol has been approved by the Ethics committee of Hangzhou cancer hospital. The ethics number is HZCH-2020-030.
\end{abstract}

Trial registration: clinicaltrials.gov, NCT04636593. Registered 19 November 2020 - Retrospectively registered Keywords: Almonertinib, EGFR-TKI, Radiotherapy, Locally advanced NSCLC, Radiation pneumonitis

\footnotetext{
* Correspondence: bingxia_hzch@163.com; zdsymsl0807@zju.edu.cn 'Department of Thoracic Oncology, Key Laboratory of Clinical Cancer Pharmacology and Toxicology Research of Zhejiang Province, Affiliated Hangzhou Cancer Hospital, Zhejiang University School of Medicine, Hangzhou 310002, People's Republic of China

Full list of author information is available at the end of the article
}

(c) The Author(s). 2021 Open Access This article is licensed under a Creative Commons Attribution 4.0 International License, which permits use, sharing, adaptation, distribution and reproduction in any medium or format, as long as you give appropriate credit to the original author(s) and the source, provide a link to the Creative Commons licence, and indicate if changes were made. The images or other third party material in this article are included in the article's Creative Commons licence, unless indicated otherwise in a credit line to the material. If material is not included in the article's Creative Commons licence and your intended use is not permitted by statutory regulation or exceeds the permitted use, you will need to obtain permission directly from the copyright holder. To view a copy of this licence, visit http://creativecommons.org/licenses/by/4.0/ The Creative Commons Public Domain Dedication waiver (http://creativecommons.org/publicdomain/zero/1.0/) applies to the data made available in this article, unless otherwise stated in a credit line to the data. 


\section{Background}

Lung cancer is the leading cancer both worldwide and in China [1]. Non-small cell lung cancer (NSCLC) accounts for approximately $85 \%$ of all lung cancer cases and 30\% of NSCLC patients are in stage III. Currently, the standard treatment for local advanced NSCLC is still doublet platinum-based therapy combined with radiotherapy, regardless of the EGFR mutation status. However, the treatment outcome is unsatisfied with an 5-year survival rate of less than $30 \%$ [2].

Many efforts including escalation of radiation dose, adjustment of chemotherapy regimen or sequence have been made to improve the outcome of local advanced NSCLC, but little progress was made. The PACIFIC study demonstrated maintenance immunotherapy with PD-L1 inhibitor durvalumab after chemoradiotherapy exerted large survival benefit, with median progressionfree survival (PFS) of 16.8 months and 4-year overall survival of $49.6 \%$, comparing to median PFS of 5.6 months and 4-year OS of $35.3 \%$ in the placebo group [3, 4]. However, subgroup analysis showed that patients with EGFR mutations might not benefit from maintenance immunotherapy.

For stage IV EGFR-mutant NSCLC, EGFR tyrosine kinase inhibitors (EGFR-TKIs) are the first-line treatment option. FLAURA study showed osimertinib prolonged mPFS to 18.9 months in treatment-naïve EGFR-mutant NSCLC patients. However, the value of EGFR-TKI in local advanced NSCLC is unknown. RECEL study was a randomized phase 2 trial comparing erlotinib and etoposide/ cisplatin with concurrent radiotherapy for patients with stage IIIA/B unresectable advanced NSCLC with activating EGFR mutation. It showed the median PFS in firstgeneration EGFR-TKI combined with thoracic radiotherapy (24.5 months) was significantly improved compared with chemoradiotherapy (9.0 months) [5]. It suggests the potential value of concurrent EGFR-TKI with radiotherapy.

Another concern is that concurrent TKI and radiotherapy could increase the incidence of radiation pneumonitis. Previous studies showed that the incidence of radiation pneumonitis in combination radiotherapy with firstgeneration TKI was as high as $40 \%$, and grade $\geq 3$ pneumonitis rate reached 20\% [6]. Severe radiation pneumonitis might counteract the benefit of EGFR-TKI and radiotherapy. Therefore, it is essential to explore the safety of TKIradiotherapy combination therapy.

Almonertinib, HS-10296, is a third-generation EGFRTKI developed by Hansoh pharmaceutical company in China. It can irreversibly bind to mutated EGFR, specifically to T790M, exon $21 \mathrm{~L} 858 \mathrm{R}$, and exon 19 deletion. At the 2020 AACR annual meeting, Lu et al. presented the preliminary results of APOLLO study, a phase 2 trial [7]. All patients progressing on prior EGFR-TKI treatment and harboring T790M mutation received almonertinib at a dose of $110 \mathrm{mg}$ per day. The object response rate (ORR) and disease control rate (DCR) were 68.9 and $93.4 \%$. The median PFS was 12.3 months. The incidence of grade 3 or higher adverse events (AEs $\geq 3$ ) was 15.6\%. The most common AEs were increased creatine phosphokinase, skin rash, increased aspartate aminotransferase, increased alanine aminotransferase et al. Notably, there was no interstitial lung disease reported. China Food and Drug Administration has therefore approved almonertinib for EGFR-sensitive-mutation or T790M mutation NSCLC patients progressing to prior EGFRTKIs.

There are few knowledges about the safety of third generation EGFR-TKI combined with thoracic radiotherapy. This study aims to explore the safety of concurrent radiotherapy with almonertinib in EGFR-mutant local advanced NSCLC patients, most importantly the incidence of radiation pneumonitis.

\section{Methods and design Study design}

The study is designed as a multi-center phase 2 study to evaluate the safety of concurrent radiotherapy with almonertinib in EGFR-mutated locally advanced NSCLC patients. Previous studies have demonstrated higher lung V20 correlated with higher the probability of radiation pneumonitis [8]. In order to reduce the influence of large PTV on radiation pneumonitis, patients with lung $\mathrm{V} 20 \geq 28 \%$ were enrolled in induction group ( $\operatorname{arm~A}$ ), which almonertinib was given for 2 months followed by concurrent radiotherapy; patients with lung V $20<28 \%$ were enrolled in concurrent group (arm B), which almonertinib was given concurrent with thoracic radiotherapy (Fig. 1).

\section{Primary endpoint}

The incidence of radiation pneumonitis ( $\geq$ grade 3 ) within 6 months post-radiotherapy.

\section{Secondary endpoints \\ - Local control rate \\ - Progression-free survival \\ - Overall survival}

\section{Inclusion criteria}

- Histologically or cytologically confirmed NSCLC.

- Inoperable stage III according to the eighth edition of TNM classification system.

- Blood or tissue EGFR detection harboring exon 19 deletion or L858R mutation.

- Forced expiratory volume in $1 \mathrm{~s}>0.75 \mathrm{~L}$.

- Age $\geq 18$ years old.

- Eastern Cooperative Oncology Group (ECOG) performance status $(\mathrm{PS}) \leq 2$. 


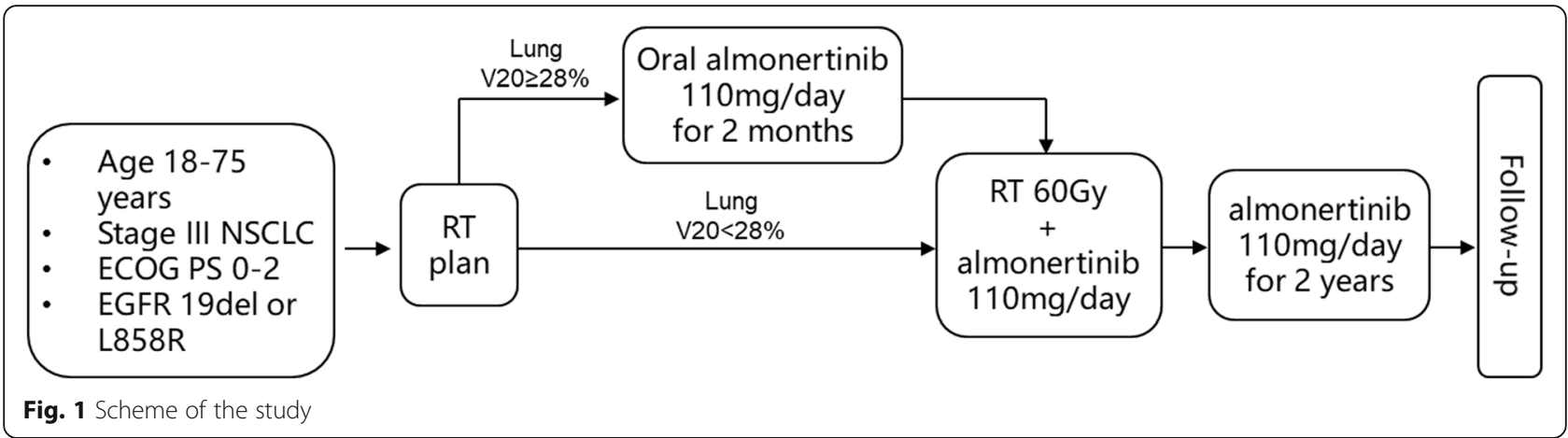

. Estimated survival period $\geq 6$ months.

- Signed the informed consent form.

\section{Exclusion criteria}

- Patients who received prior anti-tumor treatments, including chemotherapy, immunotherapy or EGFRTKIs.

- If lung $\mathrm{V} 20 \geq 28 \%$ even after 2-month induction almonertinib.

- Contraindications to radiotherapy.

- Other malignant tumors within 5 years (except for non-melanoma skin cancer and cervical cancer).

- Any medical or non-medical reasons prevent the patient from continuing to participate in the research.

- Use of any drugs or substances known to be strong or moderate inhibitors or inducers of CYP3A4 within 30 days prior to study.

- Pregnancy or breastfeeding.

\section{Treatment planning}

All enrolled patients underwent positioning CT scans before the initial treatment, a radiotherapy plan was made for each patient, and the lung V20 of each patient was calculated. Patients with lung V20 $\geq 28 \%$ were enrolled in Arm A: Almonertinib was administered for 2 months followed by almonertinib combined with radiotherapy; patients with lung V $20<28 \%$ were enrolled in Arm B: almonertinib was administered concurrent with radiotherapy. Almonertinib will be administered oral $110 \mathrm{mg} /$ day for 2 years or until disease progression or intolerable toxicity.

\section{Radiotherapy}

4D-CT with intravenous contrast should be used for all patients. Gross tumor volume (GTV) is defined as volume of the primary tumor mass and nodal diseases. Involved field radiotherapy is adopted in this study, while elective nodal irradiation is not permitted. The clinical target volume (CTV) will consist of the GTV and approximately a $0.5 \mathrm{~cm}$ margin for microscopic extension.
The plan target volume (PTV) will consist of the CTV and approximately a $0.5 \mathrm{~cm}$ margin for patient movement and setup uncertainties.

Radiotherapy will be delivered at least intensity modulated radiotherapy (IMRT). Radiotherapy dose is set to $60 \mathrm{~Gy} \pm 10 \%$. The definition target volume refers to ICRU50 and ICRU62.

Dose limits for normal tissues and organs are as follows: mean lung dose $<20 \mathrm{~Gy}$ and/or V20 $<28 \%$; mean esophageal dose $<34 \mathrm{~Gy}$; heart V $50<25 \%$, V $30 \leq 50 \%$ and V $45<35 \%$.

Cone-beam computed tomography $(\mathrm{CBCT})$ or other position verification techniques are required to verify the accuracy of patient position every week.

Interruption of radiotherapy: Interruption of radiotherapy should be avoided as much as possible. The following situations can be suspended radiotherapy: 1) The absolute number of neutrophils is less than $1.0 \times 10^{9} / \mathrm{L} ; 2$ ) Platelets are less than $\left.50 \times 10^{9} / \mathrm{L} ; 3\right) \geq 2$ radiation pneumonitis; 4) Diarrhea (water samples per day Diarrhea $\geq 7$ times), those who are ineffective after antidiarrheal treatment; 5) Weight loss $\geq 20 \%$; 6) Any unexpected adverse effects that might or might not be related to the treatment should be determined by investigators.

Recovery of radiotherapy: Continue treatment until adverse effects improve to Grade $\leq 2$, except that radiation pneumonitis should improve to Grade $\leq 1$.

Termination of radiotherapy: 1) After active management, the adverse effects have not improved to Grade $\leq$ 2 ; 2 ) The interruption of radiotherapy due to any reason is more than 10 days; 3 ) The investigator considers that it is necessary to terminate radiotherapy; 4) The patient requests to terminate radiotherapy.

\section{Safety monitoring and tumor evaluation}

All lesions must be recorded during the screening period and re-evaluated in each subsequent tumor evaluation. Tumor will be evaluated during screening, before drug administration and radiotherapy, and follow-up every 8 weeks after the start of the study. 
Medical history, demographic data, complete physical examination, vital signs, ECOG score, and laboratory tests will be recorded at each follow-up. Adverse effects will be evaluated according to National Cancer Institute Common Terminology Criteria for Adverse Events version 5.0 (NCI CTCAE 5.0).

\section{Statistical analysis}

A Simon's Two-Stage design is used in this study. The null hypothesis of the trial is that the 6-month pneumonitis-free rate of grade $\geq 3$, was less or equal to $75 \%$ versus alternative of at least $90 \%$. In this study, $n=$ 43 patients are needed for testing the null hypothesis at a one-sided significance level of $\alpha=0.05$ with a probability of $1-\beta=0.8$. At the first stage, if 5 of the 22 patients experienced grade $\geq 3$ radiation pneumonitis, then the study is stopped for futility.

All data will be reviewed by an independent Data Monitoring Committee (IDMC).

\section{Discussion}

The main purpose of this study is to explore the safety of third-generation EGFR-TKI almonertinib combined with radiotherapy for stage III NSCLC patients. The incidence of radiation pneumonitis is one of the most important indicators for this study.

Third-generation EGFR-TKI could increase radiosensitivity in EGFR-mutant NSCLC. Yu et al. showed thirdgeneration EGFR-TKI could reduce G2/M-phase cell cycle arrest and block IR-induced DNA DSB repair [9]. $\mathrm{Ma}$ et al. found that third-generation EGFR-TKI increased sensitivity to radiation by delaying DNA damage repair after irradiation and inducing apoptosis by the EGFR signaling pathway [10]. However, previous studies have showed that EGFR-TKI combined with radiotherapy can also increase the incidence of radiation pneumonitis. The EGFR signaling pathway is the most widely studied growth factor receptor. It is universal in cells and has a variety of signal transduction functions. EGFR is widely distributed in alveolar epithelium, submucosal glands, bronchial epithelial basal layer and endothelium in the respiratory system. EGFR pathway plays a critical role in repairing airway damage, promoting epithelial cell migration and proliferation. When the lung tissue is injured by radiotherapy, the EGF signaling pathway can promote the proliferation of alveolar epithelial cells to repair the integrity of the alveolar wall, thereby repairing lung injury [11]. EGFR-TKI could interferes with the EGF signaling pathway and affect the repair of lung tissue. The study of Zheng et al. showed that the incidence of radiation pneumonitis in combination radiotherapy with first-generation TKI was as high as $40 \%$, and grade $\geq 3$ pneumonitis rate reached 20\% [6]. RECEL study also showed grade $\geq 3$ radiation pneumonitis was
$16.7 \%$ in the EGFR-TKI concurrent group [12]. In fact, first-generation EGFR-TKI could not only inhibit mutant EGFR, but also potently inhibit wild-type EGFR. To the contrary, third-generation EGFR-TKI have low selectivity for wild-type EGFR, which might explain its low incidence of interstitial pneumonia [13].

Almonertinib is a third-generation EGFR-TKI. APOLLO study showed almonertinib had a favorable safety profile with no interstitial pneumonia occurred in the included patients. While the safety of almonertinib concurrent with thoracic radiotherapy is still unknown. Jia et al. reported an especially high rate of grade 2 or worse RP in patient treated with combination thoracic radiotherapy and osimertinib. Seven of 11 patients (63.6\%) experienced grade 2 or worse radiation pneumonitis and 5 (45.4\%) exhibited grade 3. Of course, Jia's study was a retrospective study with small sample size and, bias might influence the results. Therefore, a welldesigned prospective study is needed to explore the safety of third-generation EGFR-TKI combined with thoracic radiotherapy for locally advanced NSCLC patients.

\section{Abbreviations \\ AEs: Adverse events; CBCT: Cone-beam computed tomography; \\ CTCAE: Common terminology criteria; CTV: Clinical target volume; \\ DCR: Disease control rate; EGFR: Epidermal growth factor receptor; \\ GTV: Gross tumor volume; ICRU: International commission on radiation units \\ \& measurements; IMRT: Intensity modulated radiotherapy; NCl: National cancer institute; NSCLC: Non-small cell lung cancer; ORR: Object response rate; OS: Overall survival; PFS: Progression-free survival; PTV: Plan target volume; TKl: Tyrosine kinase inhibitor}

\section{Acknowledgements}

We thank the colleagues of the Department of Oncology, Hangzhou Cancer Hospital for the contribution in this study.

\section{Authors' contributions}

Writing original draft and editing: LCZ, JFL; Methodology and data acquisition: CLZ, ZCZ, JFW, LY, CZR, BX, ZPY; data analyses and interpretation: LCZ; study concept and design, project administration: BX, SLM; Manuscript revision: $L C Z, B X, S L M$. All authors read and approved the final manuscript.

\section{Funding}

This study was supported by grants from Huilan Welfare Foundations (HL-HS2020-84-2). The study protocol has been reviewed by the external, non-commercial funding body. The funders had no role in study design, data collection and analysis, decision to publish, or preparation of the manuscript.

\section{Availability of data and materials}

The datasets used and/or analyzed during the current study are available from the corresponding author on reasonable request.

\section{Declarations}

Ethics approval and consent to participate

All procedures performed in studies involving human participants were in accordance with the ethical standards of the national research committee and with the 1964 Helsinki declaration and its later amendments or comparable ethical standards. The Scientific Research Board of Hangzhou Cancer Hospital approved this study ( $\mathrm{HZCH}-2020-030)$. Administrative permissions were obtained to access the data used in this study. All patients signed the informed consent. 


\section{Consent for publication}

Not Applicable.

\section{Competing interests}

The authors have stated that they have no conflicts of interest.

\section{Author details}

'Department of Thoracic Oncology, Key Laboratory of Clinical Cancer Pharmacology and Toxicology Research of Zhejiang Province, Affiliated Hangzhou Cancer Hospital, Zhejiang University School of Medicine, Hangzhou 310002, People's Republic of China. 'Department of Radiotherapy, The First Affiliated Hospital of Wenzhou Medical University, Wenzhou 325100 People's Republic of China. ${ }^{3}$ Department of Radiotherapy, Ningbo Medical Center Lihuili Hospital, Ningbo 315100, People's Republic of China. ${ }^{4}$ Department of Radiotherapy, Shaoxing People's Hospital (Shaoxing Hospital, Zhejiang University School of Medicine), Shaoxing 312000, People's Republic of China. ${ }^{5}$ Department of Pulmonary \& Critical Care Medicine, Shulan (Hangzhou) Hospital, Affiliated to Zhejiang Shuren University Shulan International Medical college, Hangzhou 310022, People's Republic of China. ${ }^{6}$ Department of Radiotherapy \& Chemotherapy, Hwa Mei Hospital, University of Chinese Academy of Sciences, Ningbo 315010, People's Republic of China. ${ }^{7}$ Department of Oncology, Affiliated Hospital of Jiaxing University, The First Hospital of Jiaxing, Jiaxing 314000, People's Republic of China. ${ }^{8}$ Department of Oncology, Jiande Second People's Hospital, Hangzhou 311604, People's Republic of China. ${ }^{9}$ Department of Cancer Medical Center, Affiliated Xiaoshan Hospital, Hangzhou Normal University, Hangzhou 311201, People's Republic of China.

Received: 20 January 2021 Accepted: 28 April 2021

Published online: 07 May 2021

\section{References}

1. Chen W, Zheng R, Baade PD, Zhang S, Zeng H, Bray F, Jemal A, Yu XQ, He J. Cancer statistics in China, 2015. CA Cancer J Clin. 2016;66(2):115-32.

2. Howlader N, Noone AM, Krapcho M, Miller D, Brest A, Yu M, Ruhl J, Tatalovich Z, Mariotto A, Lewis DR, Chen HS, Feuer EJ, Cronin KA. SEER Cancer Statistics Review, 1975-2017. Bethesda: National Cancer Institute; 2020. https://seer.cancer.gov/csr/1975 2017/.

3. Antonia SJ, Villegas A, Daniel D, Vicente D, Murakami S, Hui R, et al. Overall survival with Durvalumab after Chemoradiotherapy in stage III NSCLC. N Engl J Med. 2018;379(24):2342-50. https://doi.org/10.1056/NEJMoa1809697.

4. Faivre-Finn C, Vicente D, Kurata T, Planchard D, Paz-Ares L, Vansteenkiste J, et al. LBA49 Durvalumab after chemoradiotherapy in stage III NSCLC: 4-year survival update from the phase III PACIFIC trial. Ann Oncol. 2020;31:S1178-9. https://doi.org/10.1016/j.annonc.2020.08.2281.

5. Xing L, Wu G, Wang L, Li J, Wang J, Yuan Z, et al. Erlotinib vs etoposide/cisplatin with radiotherapy in unresectable stage III epidermal growth factor receptor mutation-positive non-small-cell lung cancer: a multicenter, randomized, open-label, phase 2 trial. Int J Radiat Oncol Biol Phys. 2020;109(5):1349-58. https://doi.org/10.1016/j.jjrobp.2020.11.026.

6. Zheng L, Wang Y, Xu Z, Yang Q, Zhu G, Liao XY, et al. Concurrent EGFR-TK and thoracic radiotherapy as first-line treatment for stage IV non-small cell lung Cancer harboring EGFR active mutations. Oncologist. 2019;24(8):1031e1612. https://doi.org/10.1634/theoncologist.2019-0285.

7. CT190 - A multicenter, open-label, single-arm, phase II study: The third generation EGFR tyrosine kinase inhibitor almonertinib for pretreated EGFR T790M-positive locally advanced or metastatic non-small cell lung cancer (APOLLO). In: American Association for Cancer Research: 2020; 2020.

8. Hanania AN, Mainwaring W, Ghebre YT, Hanania NA, Ludwig M. Radiationinduced lung injury: assessment and management. Chest. 2019;156(1):15062. https://doi.org/10.1016/j.chest.2019.03.033.

9. Wang N, Wang L, Meng X, Wang J, Zhu L, Liu C, et al. Osimertinib (AZD9291) increases radio-sensitivity in EGFR T790M non-small cell lung cancer. Oncol Rep. 2019;41(1):77-86. https://doi.org/10.3892/or.2018.6803.

10. Wu S, Zhu L, Tu L, Chen S, Huang H, Zhang J, et al. AZD9291 increases sensitivity to radiation in PC-9-IR cells by delaying DNA damage repair after irradiation and inducing apoptosis. Radiat Res. 2018;189(3):283-91. https:// doi.org/10.1667/RR14682.1.

11. Aida S, Tamai S, Sekiguchi S, Shimizu N. Distribution of epidermal growth factor and epidermal growth factor receptor in human lung: immunohistochemical and immunoelectron-microscopic studies. Respiration. 1994;61(3):161-6.

12. Xing L, Wu G, Wang L, Li J, Wang J, Yuan Z, Chen M, Xu Y, Fu X, Zhu Z et al: Erlotinib vs etoposide/cisplatin with radiotherapy in unresectable stage III epidermal growth factor receptor mutation-positive non-small-cell lung cancer: A multicenter, randomized, open-label, phase 2 trial. Int J Rad Oncol Biol Phys 2020.

13. Wang S, Cang S, Liu D. Third-generation inhibitors targeting EGFR T790M mutation in advanced non-small cell lung cancer. J Hematol Oncol. 2016; 9(1):34. https://doi.org/10.1186/s13045-016-0268-z.

\section{Publisher's Note}

Springer Nature remains neutral with regard to jurisdictional claims in published maps and institutional affiliations.
Ready to submit your research? Choose BMC and benefit from:

- fast, convenient online submission

- thorough peer review by experienced researchers in your field

- rapid publication on acceptance

- support for research data, including large and complex data types

- gold Open Access which fosters wider collaboration and increased citations

- maximum visibility for your research: over $100 \mathrm{M}$ website views per year

At BMC, research is always in progress.

Learn more biomedcentral.com/submissions 